\title{
Pancreatic Neuroendocrine Tumor presenting as a diffuse pancreatic enlargement, case report and review of literature
}

\author{
Faeze Salahshour ${ }^{1,4}$, Reza Taslimi ${ }^{2}$, Najme-Sadat Moosavi ${ }^{1 *}$, Niloofar Ayoobi Yazdi ${ }^{1,4}$, \\ Mohsen Esfandbod ${ }^{3}$ \\ 1. Department of Radiology, Advanced diagnostic and interventional radiology research center (ADIR), Imam Khomeini Hospital, Tehran \\ University of Medical Sciences, Tehran, Iran \\ 2. Department of Gastroenterology, Imam Khomeini Hospital, Tehran University of Medical Sciences, Tehran, Iran \\ 3. Department of Oncology and Hematology, Imam Khomeini Hospital, Tehran University of Medical Sciences, Tehran, Iran \\ 4. Liver Transplantation Research Center, Imam-Khomeini Hospital, Tehran University of Medical Sciences (TUMS), Tehran, Iran \\ * Correspondence: Najme-sadat Moosavi MD-MPH, Emam Khomeini Hospital, Boulvar Keshavarz street, Tehran, Iran \\ (근.moosavi@yahoo.com)
}

Radiology Case. 2021 Jan; 15(1):11-20 :: $\quad$ DOI: $10.3941 /$ jrcr.v15i1.3822

\begin{abstract}
Pancreatic neuroendocrine tumors are rare neoplasms that comprise $1-2 \%$ of all pancreatic tumors. However, they are the second most common solid pancreatic neoplasms. They have a wide range of imaging appearances and they can show common to very rare imaging presentations. Most of the time they are solitary well-marginated enhancing solid mass arising in a certain aspect of the pancreas. We present a case report of a 41-year-old female who underwent clinical work-up for abdominal pain, loss of appetite and weight loss for the past year. Ultrasound, computed tomography, and magnetic resonance imaging show diffuse homogenous pancreatic enlargement without contour deformity or a focal mass. Lymphoma and autoimmune pancreatitis were suggested based on imaging findings but IGg4 level and other lab data were normal. Endoscopic ultrasonography confirmed the diffuse enlargement of the pancreas without peripheral structures involvement. The pathological results of multiple fine-needle aspiration biopsy from all parts of the enlarged pancreas revealed a low-grade neuroendocrine tumor.
\end{abstract}

\section{CASE REPORT}

\section{CASE REPORT}

A 41-year-old female patient with a medical history significant for Hashimoto's thyroiditis presented with nonspecific abdominal pain that sometimes radiates into the back, loss of appetite and weight loss of 5 kilograms for the past year. There is no history of nausea, vomiting, diarrhea, melena, or bleeding. Physical examination was normal. Laboratory data were normal except for elevated CA19-9 (247 units per milliliter with a normal range of $0-37$ in a healthy person).

In the course of workup, an abdominal ultrasound (US) was performed. A marked pancreatic enlargement was found with mild heterogeneous echo-pattern and no obvious focal mass or calcification. 
The contrast-enhanced computed tomography (CECT) of the abdomen showed diffuse pancreatic enlargement with normal and homogenous enhancement without obvious focal occupying lesion or peripancreatic infiltration. The main pancreatic duct had a normal appearance. There was no evidence of extra or intrahepatic bile duct dilatation and no peripancreatic or celiac enlarged lymph nodes (Figure 1).

The magnetic resonance imaging (MRI) showed diffuse pancreatic enlargement with hypo signal intensity in T1weighted images (T1WI) and iso signal intensity in T2weighted images (T2WI) compared to the liver (Figures 2 and 4). After contrast administration, diffuse homogenous enhancement in pancreatic phase was observed (Figure 3). There were few tiny cysts within the tail of the pancreas. Pancreatic and biliary ducts were unremarkable.

In this step, the differential diagnosis included autoimmune pancreatitis, lymphoma and other infiltrative pancreatic processes such as amyloidosis, sarcoid, hemochromatosis, granulomatous diseases, or much less likely a primary or metastatic malignancy.

Endoscopic ultrasonography (EUS) confirmed the diffuse enlargement of the pancreas without peripheral structures involvement. During EUS, fine needle aspiration biopsy (EUS-FNA) was performed from the head, body, and tail of the pancreas. The pathological results of specimens revealed a neuroendocrine tumor within all parts of the pancreas. In immunohistochemistry (IHC) diffuse expression of synaptophysin and chromogranin A was reported with KI-67 proliferative index $<1 \%$ which is compatible with G1(welldifferentiated) neuroendocrine tumor according to World Health Organization (WHO) classification.

After initial histological confirmation, whole body indium111-octreotide scintigraphy with single-photon emission computed tomography (SPECT) was done for staging in another center, which demonstrated somatostatin positive tumoral involvement of the entire pancreas.

The patient underwent surgery for pancreaticoduodenectomy. The pathological results showed the diffuse pancreatic neuroendocrine tumor (PNET) within the entire pancreas (Figure 5). IHC was compatible with a well-differentiated neuroendocrine tumor grade 1.

\section{DISCUSSION}

\section{Etiology \& Demographics:}

Pancreatic neuroendocrine tumors are rare neuroendocrine neoplasms with a reported incidence of $<1$ per 100,000 and account for about $1-2 \%$ of all pancreatic neoplasms. However, they are the second most common solid pancreatic neoplasms after the pancreatic ductal adenocarcinoma [1]. There are no proved differences in the epidemiology of PNETs regarding race, sex or geographic area [2]. However, the incidence increases with age and peaks in the sixth and seventh decade. PNETs are classified into functional and non-functional tumors, based on the presence or absence of symptoms according to hormone production [3]. In previous studies, functional PNETS appear more frequently, but in recent studies, non-functional PNETs (NF-PNETs) comprise approximating $80 \%$ of all cases. Accidental detection of asymptomatic PNETS due to the advances in imaging techniques may have a role in this relative increase [4]. Most PNETs occur sporadically, with $1 \%-2 \%$ associated with familial syndromes, especially multiple endocrine neoplasia type 1, tuberous sclerosis, von Hippel-Lindau syndrome, and neurofibromatosis type 1 [5]. Several studies have been done on the pathogenesis of PNETs and they suggested that chromosomal abnormalities may play a role in the etiology of PNETs [2]. In the most recent WHO classification (2017), PNETs are categorized based on the proliferative activity of the tumor as measured by mitotic count and the expression of nuclear antigen Ki-67, a marker for cellular proliferation. Well-differentiated NETs can be classified as G1 tumors, when they express $<2$ mitoses/10 high power field (HPF) and $\leq 2 \%$ Ki-67 index, G2 tumors, when they express 2-20 mitoses/10 HPF and 3-20\% Ki-67 and Grade 3 tumors with $>20$ mitoses/10 HPF and $>20 \%$ Ki-67 index. Poorly differentiated NETs including neuroendocrine carcinoma which is classified as G3 tumors with $>20$ mitoses/10 HPF and $>20 \% \mathrm{Ki}-67$ index [6].

\section{Clinical \& Imaging Findings:}

NF-PNETs are still considered rare entities. They are a heterogeneous group of malignancies that are characterized by nonspecific symptoms, leading to a delayed diagnosis. Most of the symptoms such as abdominal pain, weight loss or abdominal mass are a result of mass effect. However, NFPNETs are increasingly found incidentally at imaging. The frequency of metastatic dis-ease at the time of diagnosis is reported $60 \%-80 \%$ [7]. Imaging is useful for localization of known functioning PNETs, diagnosis of NF-PNETs, and surgical planning. Different imaging modalities exist, including US, CT, MRI, somatostatin receptor scintigraphy, positron emission tomography (PET), and EUS. These techniques could be useful for locating most PNETs with a diameter of greater than $2 \mathrm{~cm}$, but usually not visualized PNETs less than $5 \mathrm{~mm}$ [8]. At the US, PNETs mostly appear as hyper-vascular well-circumscribed round or oval hypoechoic masses and depending on the tumor size they may be heterogeneous or homogeneous [9]. CT and MRI with contrast are the most common initial imaging studies in the evaluation of patients with PNETs. Typically, PNETs are best visualized during the arterial phase. They usually appear as circum-scribed hyper-vascular solid masses that rarely obstruct the pancreatic duct. Smaller lesions are usually homogeneous, and larger lesions are more likely to have heterogeneous enhancement, which could be due to areas of cystic change, necrosis, fibrosis, and calcification [10]. EUS is an excellent modality that has proved to be useful for the detection of PNETs, especially in small tumors that are not detectable by CT or MRI. Also, EUS offers the additional benefit of obtaining biopsies for diagnosis [11]. Nuclear medicine imaging including SPECT or PET. Nuclear medicine imaging could be useful to localize functioning PNET in a patient with a specific clinical syndrome, evaluate pancreatic mass for narrowing differential diagnosis, and search for metastatic disease or recurrence. In these techniques, PNETs must be low grade and contain somatostatin receptors. The most 
frequently used technique is somatostatin-receptor scintigraphy with indium111- octreotide. In-111 octreotide binds to somatostatin receptors on the cell surface. In-111 octreotide imaging involves obtaining two sets of images. The first imaging consists of SPECT of the area of interest 4 hours after intravenous injection. The second imaging takes place 18-24 hours after injection with SPECT of the same anatomic region that was imaged the previous day, followed by wholebody imaging. A combination of anatomic and functional techniques is facilitated by the hybrid scanners (SPECT/CT and PET/CT) [12]. Recently with the availability of PET imaging, somatostatin analogues have been labeled with positron-emitting isotopes, including Gallium-68, for evaluating somatostatin receptor-expressing tumors. 68GaDOTATATE PET/CT scan can dramatically improve the tumor detectability compared to In-111 octreotide scan. It can be completed in less than 2 hours, involves lower radiation exposure and added more diagnostic information and quantification capability in comparison with In-111 octreotide scan. Also, it is proven that $68 \mathrm{Ga}$-DOTATATE PET scan has a significant impact on the management of patients with neuroendocrine tumors [13].

In NETs, tumor markers are useful in diagnosis, prognosis, therapeutic evaluation, and recurrence detection. In patients with NF-PNETs, nonspecific NET tumor markers, such as chromogranin A $(\mathrm{CgA})$, serum neuron-specific enolase and pancreatic polypeptide have been used. Among these general tumor markers, $\mathrm{CgA}$ is considered the best general neuroendocrine marker and is in-creased in 50-100\% of patients with various neuroendocrine tumors [14]. The sensitivity and specificity of $\mathrm{CgA}$ in NETs are $73 \%$ and $95 \%$, respectively. $\mathrm{CgA}$ serum or plasma levels reflect tumor load and can be associated with advanced disease. $\mathrm{CgA}$ can be false negative when patients take somatostatin analog treatment or false positive when they take proton pump inhibitors or have renal failure [15].

Radiographic presentation of PNET with diffuse enlargement of the pancreas without a focal mass is a rare entity and to the best of our knowledge is only reported in four cases previously. The first report is a case with a one-month history of non-specific symptoms. Abdominal US and CECT demonstrated marked pancreatic enlargement with extensive speckled calcifications and a few cystic changes without surrounding inflammation. At the MRI enlarged pancreas was iso-intense to liver on the T1WI and slightly increased in intensity with respect to the liver on T2WI. Post contrast images show minimal enhancement in the arterial phase with progressive enhancement in the portal phase. Besides, the evidence of portal hypertension such as splenomegaly, portal vein dilatation, and esophageal varices was present at the imaging. The patient underwent laparotomy exploration and the sample of the pancreas showed a well-differentiated Islet cell neuroendocrine tumor. The liver biopsy demonstrated cirrhotic changes possibly due to polypeptide secretion by the PNETs [16].

In the second report, the patient with vague abdominal pain and diffusely enlarged pancreas with extensive parenchymal calcifications, minimal pancreatic ductal dilatation and no focal mass in imaging studies was diagnosed with chronic pancreatitis and followed clinically for several years. Because of recurrent abdominal pain, the CECT was repeated which demonstrated new hypo-attenuation masses in the pancreatic neck and tail, some of them were hypervascular in the arterial phase. There were extensive parenchymal calcifications without pancreatic atrophy. Given the suspicion of PNETs, SPECT-CT was performed which showed intense uptake of radiotracer throughout the entire pancreas. The patient underwent pancreaticoduodenectomy and sectioning of the pancreas revealed numerous well circumscribed tumoral nodules were all confined within the pancreatic parenchyma and compatible with G2 PNETs in IHC [17].

In another report, the patient was presented with a history of 2-year intermittent melena and hematemesis and evidence of esophageal varices in endoscopy. In imaging, the pancreas was markedly enlarged with a nodular appearance and multiple calcifications. Also, evidence of portal hypertension and collateral formation was seen. EUS-FNA was performed and IHC was compatible with a well-differentiated neuroendocrine tumor grade 1 . SPECT showed intense tracer up-take along the entire pancreas. The patient declined surgery and treated with octreotide and $\beta$-adrenergic blocking agent [18].

In the last report, the patient was presented with abdominal pain, nausea, and vomiting. The CECT showed diffusely enlarged pancreatic body and tail and a focal hypervascular lesion in the tail of the pancreas. SPECT showed diffuse increased uptake in an enlarged pancreas (Figure 6). Total pancreatectomy was performed. The histological sample of the pancreas showed low-grade diffuse pancreatic neuroendocrine tumor compatible with G1 PNETs in IHC [19].

Our patient was presented with non-specific abdominal pain, loss of appetite and weight loss. Abdominal US demonstrated marked pancreatic enlargement with mild heterogeneous echo pat-tern. The CECT showed diffuse pancreatic enlargement with normal and homogenous enhancement without obvious focal occupying lesion or peripancreatic infiltration. The T1WI showed diffuse pancreatic enlargement with hypo signal intensity compared to the liver and diffuse homogenous enhancement in pancreatic phase after contrast administration. There are few tiny cysts in the pancreas tail. The main pancreatic duct was unremarkable. There was no evidence of extra or intrahepatic bile duct dilatation. EUS-FNA revealed a G1 neuroendocrine tumor. SPECT demonstrated somatostatin positive tumoral lesion involving the entire pancreas. The patient underwent surgery for pancreaticoduodenectomy.

\section{Treatment \& Prognosis:}

Compared with pancreatic adenocarcinoma and functional PNETs, non-functional PNETs have an aggressive ability. They usually invade the surrounding organs and blood vessels and most of the patients are discovered with liver metastases. So nonfunctional PNETs have a poor prognosis, with a 5-year survival of $60 \%-100 \%$ in cases of localized disease, $40 \%$ for regional, and $29 \%$ for distant metastases [16]. PNETs do not tend to respond well to chemotherapy and targeted drugs and treatment of choice is surgical resection with curative intent. 
However, there are no definitive treatment plans in PNETs that present as diffuse infiltration of the pancreas.

\section{Differential Diagnosis:}

\section{1) Lymphoplasmacytic sclerosing pancreatitis (autoimmune pancreatitis):}

The most common differential diagnoses for diffuse pancreatic enlargement without clinical features or imaging findings of acute pancreatitis is autoimmune pancreatitis (AIP). Diffuse parenchymal enlargement of the pancreas is the typical feature of AIP. The pancreatic border appears featureless with effacement of the lobular contour of the pancreas. On US, diffusely enlarged pancreatic parenchyma becomes hypoechoic. On MR, the pancreas is diffusely hypointense on T1-weighted images and mildly hyperintense on T2-weighted images. The enhancement pattern of the pancreatic parenchyma on $\mathrm{CT}$ or MR often gives a helpful clue for diagnosis. There is diffusely decreased enhancement of the pancreas during the early or pancreatic phase and delayed enhancement in the late or hepatic phase of contrast enhancement. A capsule-like rim is a highly specific sign of AIP and can be seen as a halo of soft tissue attenuation surrounding the enlarged pancreas. The rim is low attenuation on CECT, and hypo signal on both T1- and T2-weighted images, and shows delayed enhancement on MR. The characteristic MRCP finding is a Diffuse or segmental narrowing of the main pancreatic duct.

Also, AIP has many extra-pancreatic involvements, most commonly biliary tract, renal and retro-peritoneal. These findings can be diagnostic when the pancreatic manifestation of AIP is atypical. AIP often associated with elevated serum IgG4 [20].

\section{2) Pancreatic Lymphoma:}

Rarely primary or secondary lymphoma present as diffuse pancreatic enlargement. On CT and MRI, the diffuse infiltration with pancreas enlargement and irregular peripancreatic fat stranding may mimic the imaging findings of acute pancreatitis. However, in these patients the typical clinical signs of acute pancreatitis are never present. Pancreas shows low signal intensity on T1- and T2-weighted images. After contrast administration usually mild to moderate homogenous enhancement is seen. As the rule, there is no calcifications, necrosis or cystic changes. Enlarged peripancreatic and periaortic lymph nodes are present. Encasement of peripancreatic vessels can be observed. Typically, there is no significant pancreatic duct dilatation [21].

\section{3) Acute pancreatitis (AP):}

The diagnosis for AP is made clinically by signs and symptoms of an acute abdomen suggestive for pancreatitis and elevation of amylase or lipase level in blood. Imaging for the diagnosis of AP might be mandatory when clinical findings suggest pancreatitis but the amylase or lipase level is not elevated, which is often seen at delayed presentation. Also, imaging is used to establish the etiology, assess the complication and stage the severity of AP.
Despite the little value of ultrasound in the diagnosis of AP it is usually the first imaging method. Identification of gallstones and biliary dilatation may help identify impacted calculus in the bile duct as the cause of AP. The US findings of AP include diffuse or focal enlargement of the pancreas, parenchymal structural changes, hypo-echogenicity of pancreas region, and peripancreatic fluid collection [22].

CECT is the best imaging method for the initial assessment and follow-up of patients with AP.

The pancreas may be normal or enlarged with a variable amount of peripancreatic fat stranding. Local edema is a common finding and may extend into the mesentery, mesocolon, and hepato-duodenal ligaments and to the peritoneal spaces. Exudate, peripancreatic fat tissue necrosis, or hemorrhage manifest as peripancreatic fluid collections. Necrosis develops between 24 and 48 hours after the onset of $\mathrm{AP}$ and is seen as nonenhancement of all or part of the gland. Pancreatic abscess is usually formed after 4-6 weeks and see as an area of low attenuation containing pus with a thick enhancing wall [23].

MRI has gained a more prominent role in the evaluation of AP over the years. Unenhanced and contrast-enhanced $\mathrm{T} 1 \neg$ weighted and fat-suppressed T2 $\neg$ weighted gradient $\neg$ echo sequences could be used. Usually, an enlarged gland with low signal on T1WI and high signal on T2WI is seen. Compared with CECT the presence of pancreatic necrosis and peripancreatic collections could be assessed with equal accuracy. MRI is better in revealing the cause of AP with high sensitivity and specificity in diagnosis choledocholithiasis and congenital anomalies of pancreas. The pancreatic duct should be evaluated on T2 weighted images or MR cholangiopancreatography (MRCP) for the presence of dilatation or disconnection [23].

There are some other rare causes of diffuse pancreatic enlargement including primary or metastatic pancreatic tumor, plasmacytoma, and pancreatic islet cell amyloidosis which had been reported in previous studies. However, there are no specific imaging findings to differentiated these entities and the diagnosis was made by excluding the common causes and evaluating the pathologic results.

According to the five cases of diffuse PNETs, there are some imaging findings which can be helpful for diagnosis. The presence of calcification and cystic changes throughout the enlarged pancreas is seen in diffuse PNETs and it is not common in other causes of pancreatic enlargement, but the absence of this finding does not exclude the diagnosis. There is no pancreatic duct involvement. On MR, the enlarged pancreas is hypointense on T1WI and slightly hyper to isointense on T2WI. On contrast-enhanced images, there is no arterial hyper enhancement which is seen in typical PNETs. There is diffusely enhancement of the pancreas during the pancreatic phase with no significant delayed enhancement in the late phase of contrast enhancement. SPECT was performed in four cases and all of them showed intense uptake of radiotracer throughout the entire pancreas. Therefore, in the evaluation of pancreatic enlargement without typical features of AP or AIP, SPECT can be useful for diagnosis of PNETs before the invasive procedures. 


\section{TEACHING POINT}

Diffuse pancreatic enlargement is a rare presentation of pancreatic neuroendocrine tumors. In the case of diffuse enlarged pancreas without typical features of acute pancreatitis, autoimmune pancreatitis or lymphoma, diffuse pancreatic neuroendocrine tumor should be considered in a differential diagnosis. Therefore, in these patients performing nuclear medicine imaging, including SPECT and PET, can be helpful before invasive procedure.

\section{REFERENCES}

1. McKenna LR, Edil BH. Update on pancreatic neuroendocrine tumors. Gland Surg. 2014; 3(4): 258-275. PMID: 25493258

2. Cynthia Ro, Wanxing Chai, Victoria E. Yu, Run Yu. Pancreatic neuroendocrine tumors: biology, diagnosis, and treatment. Chin J Cancer. 2013; 32(6): 312-324. PMID: 23237225

3. Davies K, Conlon KC. Neuroendocrine tumors of the pancreas. Curr Gastroenterol Rep. 2009;11(2):119-27. PMID:19281699

4. Dumlu E, Karakoç D, Özdemir. Nonfunctional Pancreatic Neuroendocrine Tumors: Ad-vances in Diagnosis, Management, and Controversies. Int Surg. 2015 Jun; 100(6): 1089-1097. PMID: 25590518A

5. Dixon E, Pasieka JL. Functioning and nonfunctioning neuroendocrine tumors of the pan-creas. Curr Opin Oncol. 2007;19(1):30-5. PMID: 17133109

6. Choe J, Kim KW, Kim HJ, et al. What is new in the 2017 World Health Organization Classification and 8th American Joint Committee on Cancer staging system for pancreatic neuroendocrine neoplasms? Korean J Radiol. 2019; 20:5-17. PMID: 30627018

7. Gullo L, Migliori M, Falconi M, et al. Nonfunctioning pancreatic endocrine tumors: a multicenter clinical study. Am J Gastroenterol 2003;98(11):2435-2439. PMID:14638345

8. Kazuhiro H. Recent standardization of treatment strategy for pancreatic neuroendocrine tumors. World J Gastroenterol. 2010;16(36):4519-25. PMID:20857521

9. Malagò R, D'Onofrio M, Zamboni GA, et al. Contrastenhanced sonography of nonfunc-tioning pancreatic neuroendocrine tumors. AJR Am J Roentgenol 2009;192(2):424-430. PMID:19155405

10. Buetow PC, Miller DL, Parrino TV, Buck JL. Islet cell tumors of the pancreas: clinical, radiologic, and pathologic correlation in diagnosis and localization. RadioGraphics 1997;17(2):453-472. PMID: 9084084
11. van Essen M, Sundin A, Krenning EP, Kwekkeboom DJ. Neuroendocrine tumours: the role of imaging for diagnosis and therapy. Nat Rev Endocrinol. 2014 ;10(2):102-14. PMID: 24322649

12. Kartalis N, Mucelli RM, Sundin A. Recent developments in imaging of pancreatic neuro-endocrine tumors. Ann Gastroenterol. 2015;28(2):193-202. PMID:25830417

13. Mojtahedi A, Thamake S, Tworowska I, Ranganathan D, Delpassand E. The value of 68Ga-DOTATATE PET/CT in diagnosis and management of neuroendocrine tumors compared to current FDA approved imaging modalities: a review of literature. Am J Nucl Med Mol Imaging. 2014; 4(5): 426434. PMID: 25143861

14. Eriksson B, Oberg K, Stridsberg M. Tumor markers in neuroendocrine tumors. Digestion. 2000;62 Suppl 1:33-8. PMID: 10940685

15. Modlin IM, Oberg K, Taylor A, Drozdov I, Bodei L, Kidd M. Neuroendocrine tumor bi-omarkers: current status and perspectives. Neuroendocrinology. 2014;100(4):265-77. PMID: 25300695

16. Singh R, Calhoun S, Shin M, Katz R. Pancreatic neuroendocrine tumor with atypical ra-diologic presentation. Radiol Case Rep. 2015;3(3):162. PMID:27303533

17. Naringrekar H, Vogel A, Prestipino A, Shahid H. Imitators of chronic pancreatitis: dif-fuse neuroendocrine tumour of the pancreas. BJR Case Rep. 2017;3(4):20170015. PMID:30363193

18. Santes O, Morales-Maza J, Domínguez-Rosado I. Diffuse Enlargement of the Pancreas: An Unusual Radiologic Presentation of a Pancreatic Neuroendocrine Tumor. Clin Gastro-enterol Hepatol. 2017;15(11):165-166. PMID:28624649

19. Bhargava P, Haque K, Yang Z, Sangster G. Diffuse Pancreatic Neuroendocrine Tumor: A Rare Presentation. Indian J Nucl Med. 2018;33(4):364-365.PMID: 30386066

20. Bodily KD, Takahashi N, Fletcher JG, et al. Autoimmune Pancreatitis: Pancreatic and Ex-trapancreatic Imaging Findings. AJR Am J Roentgenol. 2009;192(2):431-7. PMID: 19155406

21. Merkle EM, Bender GN, Brambs HJ. Imaging findings in pancreatic lymphoma: differen-tial aspects. AJR Am J Roentgenol.;174(3):671-5. PMID:10701607

22. Badea R. Ultrasonography of acute pancreatitis -- an essay in images. Rom J Gastroenter-ol. 2005;14(1):83-9. PMID: 1580070

23. O'Connor OJ, McWilliams S, Maher MM. Imaging of Acute Pancreatitis. AJR Am J Roentgenol. 2011;197(2): W221-5. PMID:21785045 


\section{FIGURES}

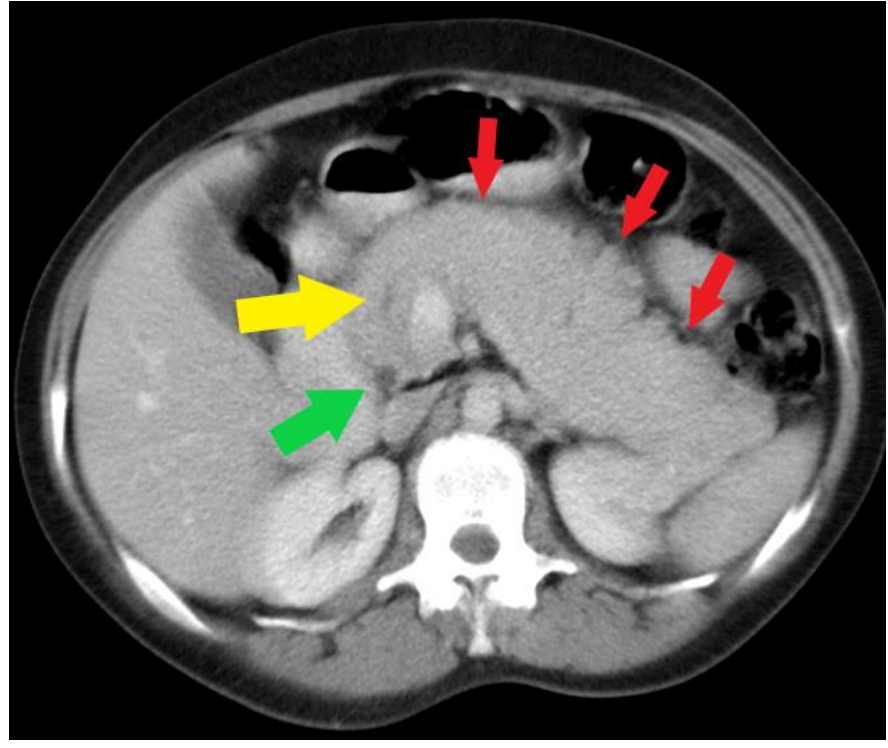

Figure 1: 41-year old female with diffuse non-functional pancreatic neuroendocrine tumor

Findings: Axial contrast-enhanced CT showing diffuse enlargement of pancreas with lobular con-tour and homogenous enhancement without calcifications or cystic changes (red arrows). Pancreatic duct (yellow arrow) and common biliary duct (green arrow) appear normal.

Technique: Axial Multi-detector CT, Portal Venous phase (100 ml Omnipaque), mA 50-200; kV 120; $8.0 \mathrm{~mm}$ slice thickness.

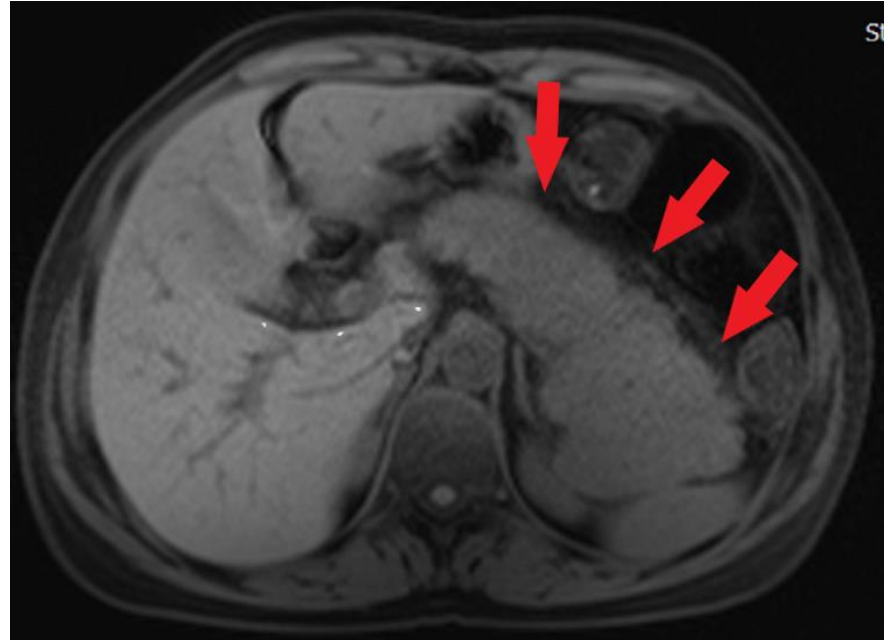

Figure 2: 41-year old female with diffuse non-functional pancreatic neuroendocrine tumor

Findings: Axial T1-weighted fat saturated MRI showing diffusely enlarged pancreas without pancreatic contour deformity or involvement of adjacent soft tissues (red arrows). The pancreas is relatively hypo signal intensity compared to the liver.

Technique: Axial T1-weighted fat saturation (3T, TR $=540$, $\mathrm{TE}=10,4 \mathrm{~mm}$ slice thickness)

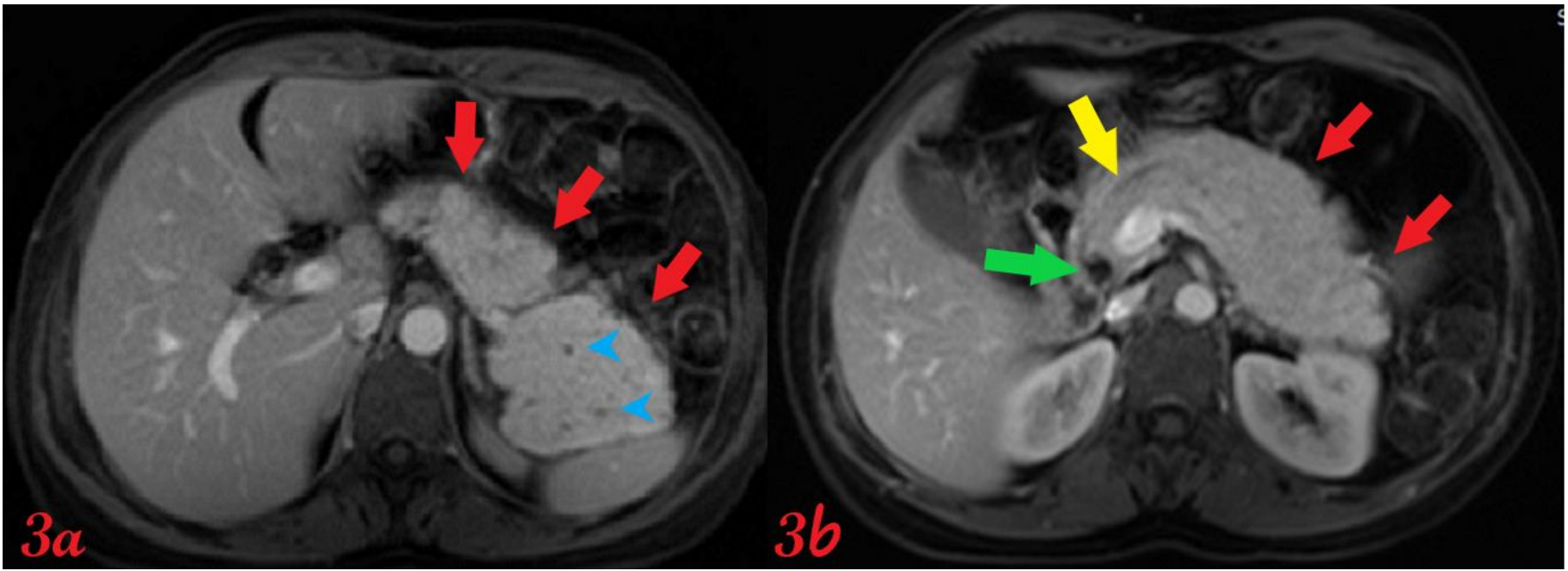

Figure 3: 41-year old female with diffuse non-functional pancreatic neuroendocrine tumor

Findings: Axial post-contrast T1-weighted fat saturated MRI in pancreatic phase showing enlarged pancreas with diffuse homogenous enhancement (red arrows). There are few tiny cysts within the tail of pancreas (blue arrowhead in 3a). Pancreatic duct (yellow arrow in $3 b$ ) and common biliary duct (green arrow in $3 b$ ) appear normal.

Technique: Axial post-contrast T1-weighted fat saturation, pancreatic phase $(3 \mathrm{~T}, \mathrm{TR}=540, \mathrm{TE}=10,4 \mathrm{~mm}$ slice thickness, $10 \mathrm{ml}$ intravenous Dotarem) 


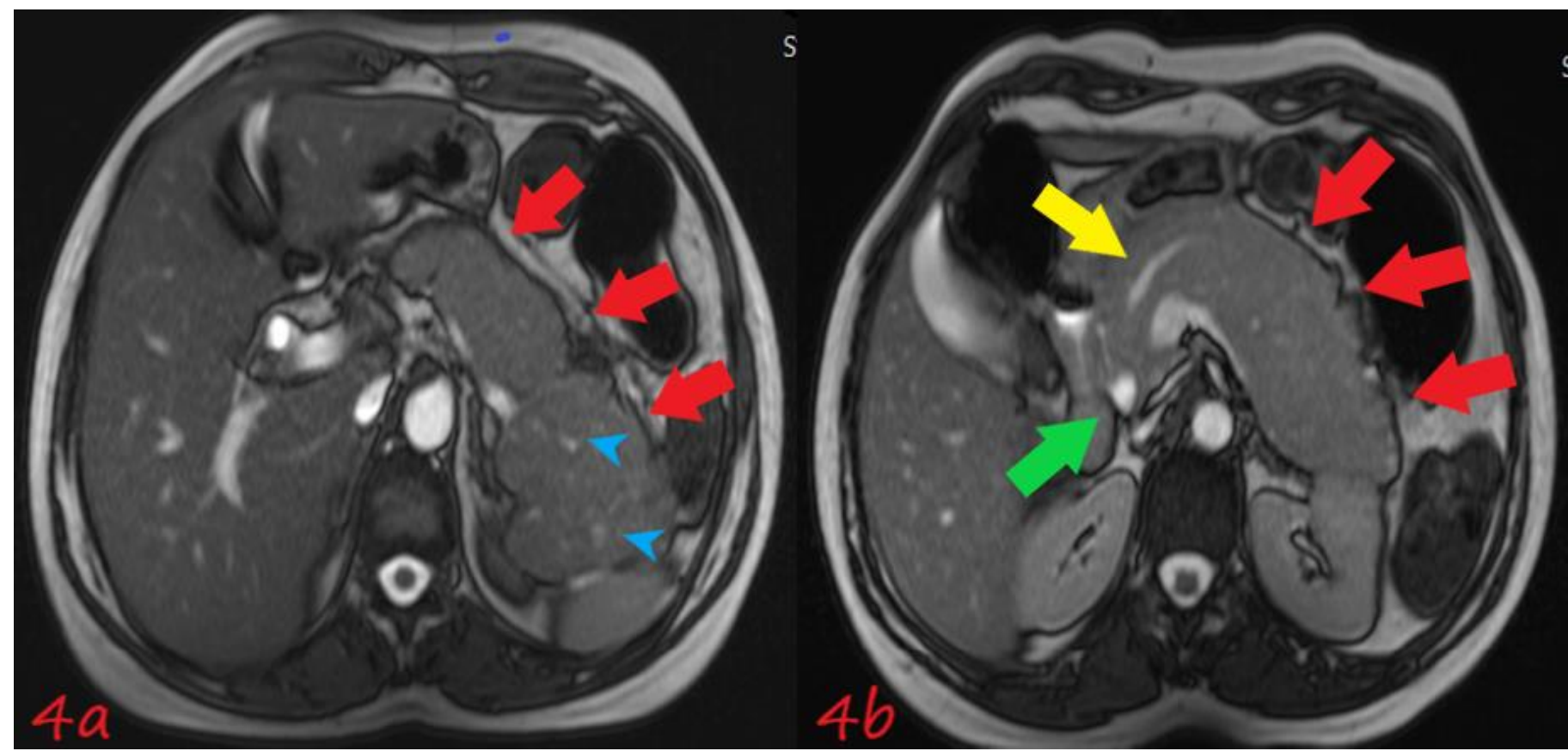

Figure 4: 41-year old female with diffuse non-functional pancreatic neuroendocrine tumor

Findings: Axial T2-weighted MRI showing diffusely enlarged pancreas without pancreatic contour deformity or involvement of adjacent soft tissues (red arrows). The pancreas is relatively iso-signal intensity compared to the liver. There are few tiny cysts within the tail of pancreas (blue arrowhead in 4a). Pancreatic duct (yellow arrow in 4b) and common biliary duct (green arrow in 4b) appear normal.

Technique: Axial T2-weighted (3T, TR $=4350, \mathrm{TE}=105,4 \mathrm{~mm}$ slice thickness)

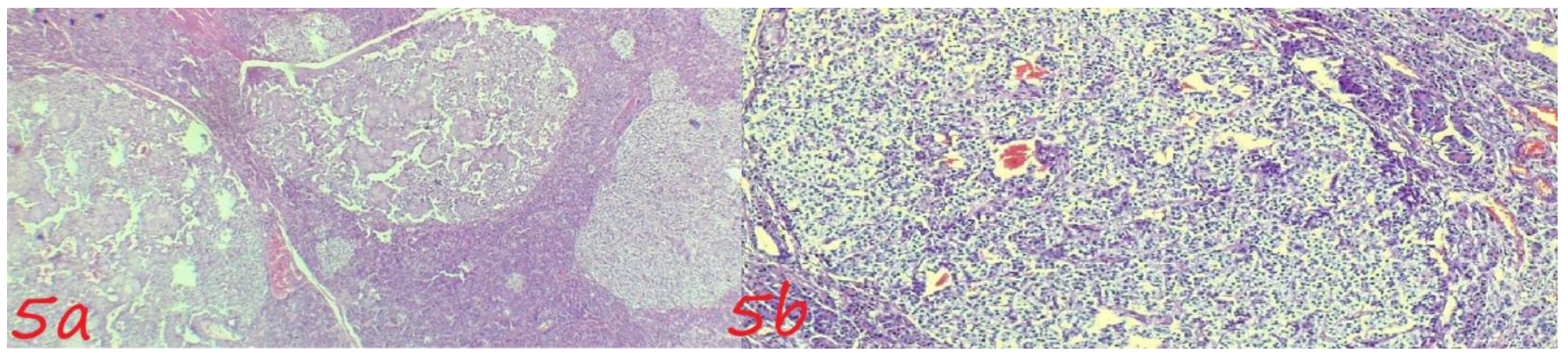

Figure 5: 41-year old female with diffuse non-functional pancreatic neuroendocrine tumor

Findings: Histopathology result obtained from surgery revealed nests of polygonal tumor cells which typically have stippled saltand-pepper chromatin and eosinophilic granular cytoplasm with plasmacytoid features 


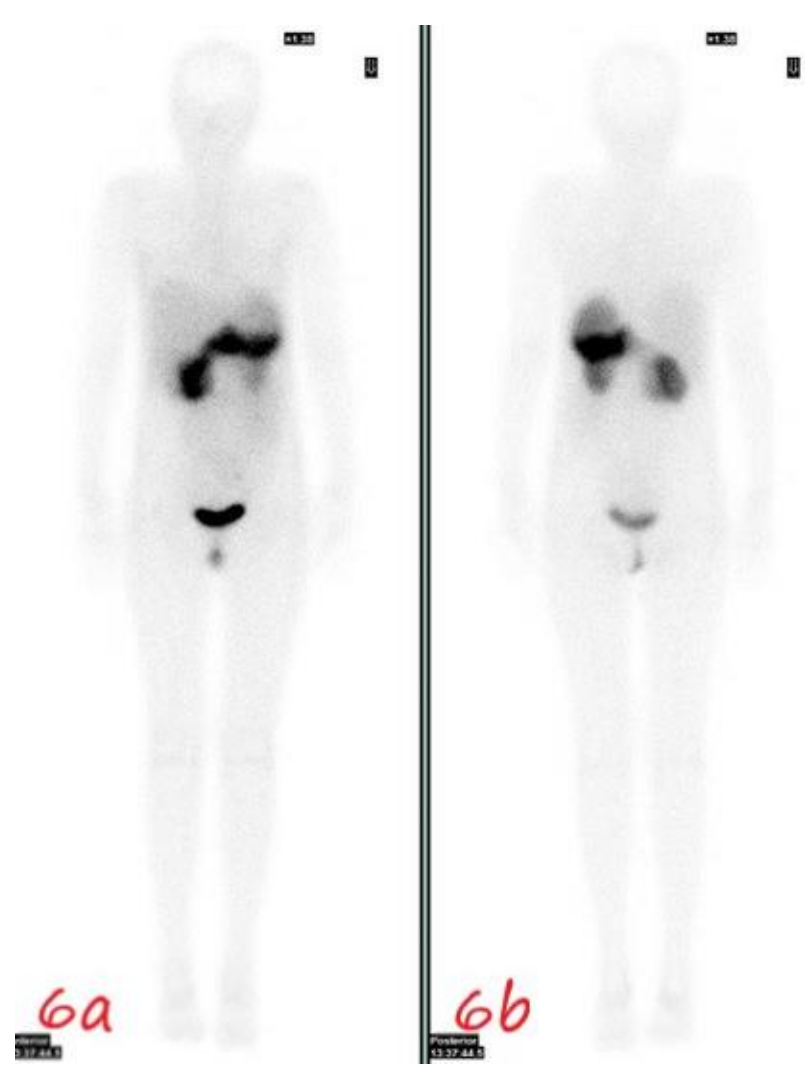

Figure 6: 41-year old female with diffuse non-functional pancreatic neuroendocrine tumor.

Findings: Diffuse increased uptake is seen in the enlarged pancreas involving the head, body and the tail of the pancreas. (copyrighted image with the permission of corresponding author)

Technique: Anterior and posterior whole-body images (a and b respectively) acquired 6 hours after IV administration of 222 $\mathrm{MBq}(6.0 \mathrm{mCi})$ of In-111 octreotide.

\begin{tabular}{|l|l|}
\hline Etiology & Possibly due to chromosomal abnormalities \\
\hline Incidence & $<1$ per 100,000 and account for about 1-2\% of all pancreatic neoplasms \\
\hline Gender ratio & None \\
\hline Age predilection & The incidence increases with age and peaks in the sixth and seventh decade \\
\hline Risk factors & $\begin{array}{l}\text { Familial syndromes, especially multiple endocrine neoplasia type 1, tuberous sclerosis, von Hippel-Lindau } \\
\text { syndrome, and neurofibromatosis type 1 }\end{array}$ \\
\hline Treatment & Mainstay treatment is the surgical excision \\
\hline Prognosis & $\begin{array}{l}\text { Nonfunctional pancreatic neuroendocrine tumors (PNETs) have poor prognosis, with a 5-year survival of } \\
60 \%-100 \% \text { in cases of localized disease, 40\% for regional, and 29\% for distant metastases }\end{array}$ \\
\hline Findings on Imaging & $\begin{array}{l}\text { We describe a case with diffuse nonfunctional PNETs } \\
\text { - Ultrasound: Diffuse pancreatic enlargement with or without calcifications } \\
\text { CECT: Diffuse pancreatic enlargement with or without calcifications or cystic changes, with diffuse } \\
\text { enhancement in pancreatic phase } \\
\text { - MRI: Diffuse pancreatic enlargement with low signal on T1- weighted image and slightly high signal } \\
\text { on T2-weighted image. Contrast images show low enhancement in arterial phase and diffuse } \\
\text { enhancement in pancreatic phase without delayed enhancement in venous phase } \\
\text { - SPECT: Intense uptake of radiotracer throughout the entire pancreas }\end{array}$ \\
\hline
\end{tabular}

Table 1: Summary table of pancreatic neuroendocrine tumor. 


\begin{tabular}{|c|c|c|c|}
\hline Differential Diagnosis & US & CECT & MRI \\
\hline $\begin{array}{l}\text { Acute } \\
\text { pancreatitis }\end{array}$ & $\begin{array}{l}\text { Hypo-echogenicity of } \\
\text { pancreas, peripancreatic } \\
\text { fluid collection, sometimes } \\
\text { gallstones and biliary } \\
\text { dilatation }\end{array}$ & $\begin{array}{l}\text { Variable amount of } \\
\text { peripancreatic fat stranding, local } \\
\text { edema, peripancreatic fluid } \\
\text { collections, pancreatic abscess or } \\
\text { necrosis }\end{array}$ & $\begin{array}{l}\text { Low signal on T1-weighted image } \\
\text { (WI) and high signal on T2WI, } \\
\text { peripancreatic collections, pancreatic } \\
\text { abscess or necrosis, may } \\
\text { demonstrate choledocholithiasis or } \\
\text { congenital anomalies of pancreas, } \\
\text { sometimes pancreatic duct dilatation } \\
\text { or disconnection }\end{array}$ \\
\hline $\begin{array}{l}\text { Autoimmune } \\
\text { pancreatitis }\end{array}$ & $\begin{array}{l}\text { Hypo-echogenicity of } \\
\text { pancreas }\end{array}$ & $\begin{array}{l}\text { Pancreatic border appears } \\
\text { featureless with effacement of the } \\
\text { lobular contour (sausage } \\
\text { pancreas), decreased } \\
\text { enhancement during the early or } \\
\text { pancreatic phase and delayed } \\
\text { enhancement in the late or } \\
\text { hepatic phase, low attenuation } \\
\text { capsule-like rim }\end{array}$ & $\begin{array}{l}\text { Hypointense on T1WI and mildly } \\
\text { hyperintense on T2WI, decreased } \\
\text { enhancement during the early or } \\
\text { pancreatic phase and delayed } \\
\text { enhancement in the late or hepatic } \\
\text { phase, hypo signal T1WI and T2WI } \\
\text { capsule-like rim with delayed } \\
\text { enhancement, diffuse or segmental } \\
\text { narrowing of the main pancreatic } \\
\text { duct }\end{array}$ \\
\hline Pancreatic lymphoma & $\begin{array}{l}\text { Hypo-echogenicity of } \\
\text { pancreas, peripancreatic } \\
\text { and periaortic } \\
\text { lymphadenopathy }\end{array}$ & $\begin{array}{l}\text { Glandular enlargement with poor } \\
\text { definition, typically uniform low } \\
\text { attenuation with minimal } \\
\text { enhancement, peripancreatic and } \\
\text { periaortic lymph node } \\
\text { enlargement, sometimes } \\
\text { encasement of the peripancreatic } \\
\text { vessels, there is no calcifications } \\
\text { or necrosis }\end{array}$ & $\begin{array}{l}\text { low signal intensity on T1- and T2- } \\
\text { weighted MR images, homogeneous } \\
\text { contrast enhancement sometimes } \\
\text { with small foci of reduced or absent } \\
\text { enhancement, peripancreatic and } \\
\text { periaortic lymph node enlargement, } \\
\text { some-times encasement of the peri- } \\
\text { pancreatic vessels, no significant } \\
\text { pancreatic duct dilatation }\end{array}$ \\
\hline $\begin{array}{l}\text { Pancreatic } \\
\text { neuroendocrine tumor }\end{array}$ & $\begin{array}{l}\text { Heterogenicity of pancreas } \\
\text { with or without } \\
\text { calcifications and cystic } \\
\text { changes }\end{array}$ & $\begin{array}{l}\text { May demonstrate multiple } \\
\text { calcifications or cystic changes, } \\
\text { diffuse enhancement in } \\
\text { pancreatic phase }\end{array}$ & $\begin{array}{l}\text { Hypointense on T1WI and slightly } \\
\text { hyperintense on T2WI, diffusely } \\
\text { enhancement of the pancreas during } \\
\text { the pancreatic phase with no } \\
\text { significant delayed enhancement in } \\
\text { the late phase, no pancreatic or } \\
\text { biliary ducts changes }\end{array}$ \\
\hline
\end{tabular}

Table 2: Differential diagnosis table for diffuse enlargement of pancreas. 


\section{ABBREVIATIONS}

AIP $=$ Autoimmune pancreatitis

$\mathrm{AP}=$ Acute pancreatitis

CECT $=$ Contrast-enhanced computed tomography

$\mathrm{Cg} \mathrm{A}=$ Chromogranin $\mathrm{A}$

EUS $=$ Endoscopic ultrasonography

FNA $=$ Fine needle aspiration biopsy

$\mathrm{HPF}=$ High power field

$\mathrm{IHC}=$ Immunohistochemistry

MRCP = MR cholangiopancreatography

MRI = Magnetic resonance imaging

NF-PNET $=$ Non-functional PNET

PET $=$ Positron-emission tomography

PNET $=$ Pancreatic neuroendocrine tumor

SPECT $=$ Single photon emission computed tomography

T1WI $=$ T1-weighted image

T2WI $=$ T2-weighted image

US = Ultrasound

WHO $=$ World Health Organization

\section{KEYWORDS}

Pancreas; Neuroendocrine tumors; Non-functional Neuroendocrine tumors; Diffuse enlargement; Computed Tomography; Magnetic Resonance Imaging

\section{ACKNOWLEDGEMENTS}

I would like to thank Dr. Peeyush Bhargava to allow us to use their patient SPECT image in this article.

\section{Online access}

This publication is online available at:

www.radiologycases.com/index.php/radiologycases/article/view/3822

\section{Peer discussion}

Discuss this manuscript in our protected discussion forum at: www.radiolopolis.com/forums/JRCR

\section{Interactivity}

This publication is available as an interactive article with scroll, window/level, magnify and more features.

Available online at www.RadiologyCases.com

Published by EduRad

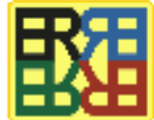

www.EduRad.org 